BULL. AUSTRAL. MATH. SOC.

\title{
A Fenchel-Rockafellar type duality theorem for maximization
}

\section{Ivan Singer}

We prove that $\sup (f-h)(E)=\sup \left(h^{*}-f^{*}\right)\left(E^{*}\right)$, where $f$ is a proper lower semi-continuous convex functional on a real locally convex space $E, h: E \rightarrow \bar{R}=[-\infty,+\infty]$ is an arbitrary functional and $f^{*}, h^{*}$ are their convex conjugates respectively. When $h=\delta_{G}$, the indicator of a bounded subset $G$ of $E$, this yields a formula for $\sup f(G)$.

\section{1 .}

A functional $f$ on a real locally convex space $E$ is said to be proper, if $f(E) \subset(-\infty,+\infty]$ and $f$ is not identically +o. For any functional $f: E \rightarrow \bar{R}=\left[-^{\infty},+\infty\right]$, the convex conjugate $f^{*}$ and the concave conjugate $f^{+}$of $f$ are the functionals on $E^{*}$ (the space of all continuous linear functions on $E$ ) defined respectively by

$$
\begin{aligned}
& f^{*}(\Phi)=\sup (\Phi-f)(E) \quad\left(\Phi \in E^{*}\right), \\
& f^{+}(\Phi)=-(-f)^{*}(-\Phi)=\inf (\Phi-f)(E) \quad\left(\Phi \in E^{*}\right) .
\end{aligned}
$$

We recall the following duality theorem of Fenchel-Rockafellar for minimization (see, for example, [2], Theorem 1, or [1], p. 68). Let $f$ and $-h$ be proper convex functionals on a real locally convex space $E$, such that one of them is continuous at some point of

$$
\{x \in E \mid f(x)<+\infty,-h(x)<+\infty\} .
$$

Then

$$
\inf (f-h)(E)=\sup \left(h^{+}-f^{*}\right)\left(E^{*}\right),
$$

Received 19 January 1979. 
and there exists $\Phi_{0} \in E^{*}$ for which the sup in (3) is attained. This theorem has various applications in optimization theory (see, for example, [3] and the references therein); for example, it yields a formula for inf $f(G)$, where $G$ is a convex subset of $E$ and $f$ is a proper convex functional on $E$, continuous at some point of $\{g \in G \mid f(g)<+\infty\}$, by taking in (3), $h=-\delta_{G}$, where $\delta_{G}$ is the indicator of the set $G$ (that is $\delta_{G}(x)=0$ if $x \in G$ and $\delta_{G}(x)=+\infty$ if $\left.x \in E \backslash G\right)$.

In the present paper we shall show, generalizing the methods of our previous paper [4], that a Fenchel-Rockafellar type duality theorem holds also for $\sup (f-h)(E)$, under a convenient assumption on $f$ and with arbitrary $h$ (Theorem $I$ below). In the particular case when $h=\delta_{G}$, the indicator of a bounded subset $G$ of $E$, this theorem yields the main quasi-lagrangian duality formula of [4] for sup $f(G)$ (Remark 3 below).

2.

We shall denote by $\Gamma_{0}(E)$ the set of all proper lower semi-continuous convex functionals on a real locally convex space $E$.

LEMMA, 1. Let $E$ be a real Zocally convex space, $f \in \Gamma_{0}(E)$ and $x_{0} \in E$. Then

$$
f\left(x_{0}\right)=\sup _{\Phi \in E^{*}}\left\{\inf (f-\Phi)(E)+\Phi\left(x_{0}\right)\right\}
$$

Proof. By (I), for any $\Phi \in E^{*}$, we have

$$
\Phi\left(x_{0}\right)-f^{*}(\Phi)=\Phi\left(x_{0}\right)-\sup (\Phi-f)(E)=\inf (f-\Phi)(E)+\Phi\left(x_{0}\right) .
$$

But since $f \in \Gamma_{0}(E)$, we have $f^{* *}\left(x_{0}\right)=f\left(x_{0}\right)$ (see, for example, [1], p. 46). Consequently,

$$
\begin{aligned}
f\left(x_{0}\right)=f^{* *}\left(x_{0}\right) & =\sup _{\Phi \in E^{*}}\left\{\Phi\left(x_{0}\right)-f^{*}(\Phi)\right\} \\
& =\sup _{\Phi \in E^{*}}\left\{\inf (f-\Phi)(E)+\Phi\left(x_{0}\right)\right\},
\end{aligned}
$$

which completes the proof of Lemma 1.

REMARK 1. In general, the sup in (4) need not be attained (since, 
obviously, it is attained for some $\Phi_{0} \in E^{*}$ if and only if $\left.\Phi_{0} \in \partial f\left(x_{0}\right)\right)$, but, if $f$ is also finite and continuous at $x_{0}$, then the sup in (4) is attained for some $\Phi_{0} \in E^{*}$.

THEOREM 1. Let $E$ be a real locally convex space, $f \in \Gamma_{0}(E)$, and $h: E \rightarrow \bar{R}=[-\infty,+\infty]$ an arbitrary functional. Then

$$
\sup (f-h)(E)=\sup \left(h^{*}-f^{*}\right)\left(E^{*}\right) \text {. }
$$

Proof $^{1}$. Assume, for the moment, that $f, h: E \rightarrow \vec{R}=[-\infty,+\infty]$ are two arbitrary functionals. Then, by (1), for any $\Phi \in E^{*}$, we have

$$
\begin{aligned}
h^{*}(\Phi)-f^{*}(\Phi) & =\sup (\Phi-h)(E)-\sup (\Phi-f)(E) \\
& =\sup (\Phi-h)(E)+\inf (f-\Phi)(E) .
\end{aligned}
$$

Assume now, a contrario, that

$$
\sup (f-h)(E)<\sup \left(h^{*}-f^{*}\right)\left(E^{*}\right) .
$$

Then there exists $\Phi_{0} \in E^{*}$ such that, using also (7),

$$
\sup (f-h)(E)<\left(h^{*}-f^{*}\right)\left(\Phi_{0}\right)=\sup \left(\Phi_{0}-h\right)(E)+\inf \left(f-\Phi_{0}\right)(E),
$$

and hence there exists also $x_{0} \in E$ such that

$$
\sup (f-h)(E)<\Phi_{0}\left(x_{0}\right)-h\left(x_{0}\right)+f\left(x_{0}\right)-\Phi_{0}\left(x_{0}\right)=f\left(x_{0}\right)-h\left(x_{0}\right),
$$

which is absurd, so (8) can not hold. Thus,

$$
\sup (f-h)(E) \geq \sup \left(h^{*}-f^{*}\right)\left(E^{*}\right) .
$$

Assume now, a contrario, that $f \in \Gamma_{0}(E)$ and

$$
\sup (f-h)(E)>\sup \left(h^{*}-f^{*}\right)\left(E^{*}\right) .
$$

Then there exists $x_{0} \in E$ such that we have, using also (7) and Lemma 1 ,

\footnotetext{
For a shorter proof, see the addendum at the end of this note.
} 


$$
\begin{aligned}
f\left(x_{0}\right)-h\left(x_{0}\right) & >\sup \left(h^{*}-f^{*}\right)\left(E^{*}\right) \\
& =\sup _{\Phi \in E^{*}}\{\sup (\Phi-h)(E)+\inf (f-\Phi)(E)\} \\
& \geq \sup _{\Phi \in E^{*}}\left\{\Phi\left(x_{0}\right)-h\left(x_{0}\right)+\inf (f-\Phi)(E)\right\} \\
& =f\left(x_{0}\right)-h\left(x_{0}\right),
\end{aligned}
$$

which is ebsurd, so (10) can not hold. This, together with (9), yields (6), completing the proof of Theorem 1 .

REMARK 2. As shown by the above proof, the inequality (9), that is, the inequality $\geq$ in (6), is valio for any twe functionals $f, h: E \rightarrow \bar{R}=[-\infty,+\infty]$. It is well known (see, for example, [1], p. 67) that the same is also true for the inequality $\geq$ in (3).

REMARK 3. In the particular case when $h=\delta_{G}$, the indicator of a bounded subset $G$ of $E$, Theorem 1 yields the following main "quasilagrangian duality theorem" of [4] (see [4], Theorem 2.1). Let $E$ be a real locally convex space, $f \in \Gamma_{0}(E)$ and $G$ a bounded subset of $E$. Then

$$
\begin{aligned}
\sup f(G) & =\sup _{\Phi \in E^{*}}\{\inf (f-\Phi)(E)+\sup \Phi(G)\} \\
& =\sup _{\Phi \in E^{*}}\{\inf (f+\Phi)(E)-\inf \Phi(G)\} .
\end{aligned}
$$

Of course, in the particular case when $G$ is a singleton $\left\{x_{0}\right\}$, this result reduces to Lemma 1 above. The sup in the right hand side of (11) (and hence of (6)) need not be attained, even when $f$ is also finite and continuous on $E$ (see [4]). Some other applications of (11) are given in [4].

ADDENDUM [Received 6 February 1979]. (1) After this paper had been submitted, Th. Precupanu has communicated to us the following simplification of the above proof of Theorem $I$.

Proof. Since $f \in \Gamma_{0}(E)$, we have $f^{* *}=f$ (see, for example, [1], p. 46). Hence 


$$
\begin{aligned}
\sup (f-h)(E) & =\sup \left(f^{* *}-h\right)(E) \\
& =\sup _{x \in E}\left\{\sup _{\Phi \in E^{*}}\left\{\Phi(x)-f^{*}(\Phi)\right\}-h(x)\right\} \\
& =\sup _{\Phi \in E^{*}}\left\{\sup _{x \in E}\{\Phi(x)-h(x)\}-f^{*}(\Phi)\right\}=\sup \left(h^{*}-f^{*}\right)\left(E^{*}\right),
\end{aligned}
$$

which completes the proof of Theorem 1. Let us observe that this argument yields also Remark 2 above, since $f \geq f^{* *}$ for any functional $f: E \rightarrow \bar{R}=[-\infty,+\infty]$.

(2) Let us also observe the following corollary of Theorem I (which is, actually, equivalent to Theorem 1), which should be compared with (3).

COROLLARY 1. Let $E$ be a real locally convex space,

$f: E \rightarrow \bar{R}=[-\infty,+\infty]$ an arbitrary functional, and $h \in \Gamma_{0}(E)$. Then

$$
\inf (f-h)(E)=\inf \left(h^{*}-f^{*}\right)\left(E^{*}\right) .
$$

Proof. By Theorem I we have

$$
\begin{aligned}
\inf (f-h)(E) & =-\sup (h-f)(E)=-\sup \left(f^{*}-h^{*}\right)\left(E^{*}\right) \\
& =\inf \left(h^{*}-f^{*}\right)\left(E^{*}\right),
\end{aligned}
$$

which completes the proof.

\section{References}

[1] Richard B. Holmes, A course on optimization and best approximation (Lecture Notes in Mathematics, 257. Springer-Verlag, Berlin, Heidelberg, New York, 1972).

[2] R.T. Rockafellar, "Extension of Fenchel's duality theorem for convex functions", Duke Math. J. 33 (1966), 81-89.

[3] Ivan Singer, "Some new applications of the Fenchel-Rockafellar duality theorem: Lagrange multiplier theorems and hyperplane theorems for convex optimization and best approximation", Nonlinear Anal. 3 (1979), 239-248. 
[4] Ivan Singer, "Maximization of lower semi-continuous convex functionals on bounded subsets of locally convex spaces. II: QuasiLagrangian duality theorems", submitted.

Institutul Nafional pentru Creafie Stiinfifică şi Tehnică, Bucureşti,

Romania;

Institutul de Matematica,

Bucureşti,

Romania. 